\title{
Associations of serum vitamins levels with bone mineral density in the different race- ethnicities US adults
}

\author{
Xiang $\mathrm{Li}^{1}$ and Xun Liü ${ }^{2^{*}}$
}

\begin{abstract}
Background: The conclusions on the associations of specific vitamin levels with bone mineral density (BMD) were controversial. Therefore, the aims of this study were to examine the associations of serum vitamins levels with BMD and the modified effect of race/ ethnicity on these associations in the US adults.

Methods: This study was from the third National Health and Nutrition Examination Survey. All participants aged $\geq 18$ years with complete data were eligible. Serum vitamins A, B9, B12, C, and E levels were assayed using the Quantaphase II Radioassay Kit (Bio-Rad). Dual-energy X-ray absorptiometry was employed to measure BMD, including femur neck and the total hip.

Results: There were 6023 participants included in the final analysis. Serum folate, vitamins A and C levels were positively associated with BMD. No significant associations of serum vitamins B12 and E levels with BMD were observed. There were positive associations of serum folate level $(\beta=0.00027$ and 0.00032 ; and $95 \%$ Cl: $0.00002-$ 0.00057 and $0.00002-0.00063$, respectively), vitamin $A$ level $(\beta=0.01132$ and 0.01115 ; and $95 \%$ Cl: $0.00478-0.01787$ and 0.00430-0.01799, respectively), and vitamin C level ( $\beta=0.00027$ and 0.00029; and 95\% Cl: 0.00012-0.00042 and 0.00013-0.00045, respectively) with BMD at femur neck and the total hip only in the Not Hispanic participants.
\end{abstract}

Conclusion: Elevated serum folate, vitamins A and C levels were associated with a higher BMD. Furthermore, sex and race/ ethnicity modified the associations of serum vitamins levels with BMD.

Keywords: Vitamin a, Serum folate, Vitamin B12, Vitamin C, Vitamin E, Bone mineral density

\section{Background}

As an important public health issue, osteoporosis is the most prevalent disease in women and the second one in men $[1,2]$. The prevalence of osteoporosis was approximately $29.9 \%$ in females aged 50 and older in America, where half of nearly nine million fractures attributed to osteoporosis occur annually [3, 4]. In the USA, there were more than 2 million newly incident fractures annually, which caused the total costs of $\$ 16.9$ billion [5]. By

\footnotetext{
*Correspondence: liliudazui@126.com

${ }^{2}$ Department of Ultrasonics, Tianjin Fifth Central Hospital, No 41 Zhejiang Road, Tianjin 300450, China

Full list of author information is available at the end of the article
}

2025, it was estimated that the total costs of osteoporotic fracture will be projected to $\$ 25.3$ billion [6]. Therefore, improving BMD and reducing bone loss are essential and critical to prevent from osteoporosis.

It is well established that there is an important role of nutritional factors in bone mineral density (BMD) [7]. A number of literatures have declared that there were significant associations of dietary factors and nutrients with bone health $[8,9]$. Previous studies found that deficiencies of $\mathrm{Ca}, \mathrm{Fe}$, vitamins $\mathrm{A}, \mathrm{K}$ and $\mathrm{D}$ were positively associated with a higher risk of osteoporosis $[8,10]$. Especially, vitamins have been identified as the main influence factors of BMD. However, the conclusions on

C C The Author(s). 2021 Open Access This article is licensed under a Creative Commons Attribution 4.0 International License, which permits use, sharing, adaptation, distribution and reproduction in any medium or format, as long as you give appropriate credit to the original author(s) and the source, provide a link to the Creative Commons licence, and indicate if changes were made. The images or other third party material in this article are included in the article's Creative Commons licence, unless indicated otherwise in a credit line to the material. If material is not included in the article's Creative Commons licence and your intended use is not permitted by statutory regulation or exceeds the permitted use, you will need to obtain permission directly from the copyright holder. To view a copy of this licence, visit http://creativecommons.org/licenses/by/4.0/ The Creative Commons Public Domain Dedication waiver (http://creativecommons.org/publicdomain/zero/1.0/) applies to the data made available in this article, unless otherwise stated in a credit line to the data. 
the associations of specific vitamins with BMD were controversial [11, 12]. Furthermore, the existed studies focused on the associations of dietary intakes and supplements of vitamins with BMD. There were few studies to examine the associations of serum vitamins levels with BMD. On the other hand, a previous study declared that race-ethnicity was a major determinant of BMD [13]. Many studies also reported that hip fracture rates differed among varied races or ethnicities, such as African-American, Hispanic, and Caucasian humans in the USA [14-16]. However, little was known on the associations of serum vitamins levels with BMD across race/ethnic groups.

In view of the above mentioned facts, the aims of this study were twofold: to examine the associations of serum vitamins levels, including vitamins A, B9, B12, C, and $\mathrm{E}$, with BMD in US adults; and to examine how sex and race-ethnicity modified the associations of serum vitamins levels with BMD. As a result, this study would provide additional evidence to improve BMD and prevent from osteoporosis, as well as assist individuals to benefit from faster screening for osteoporosis.

\section{Methods}

All authors declared that all methods in this study were carried out in accordance with relevant guidelines and regulations.

\section{Study design}

This study was conducted under the National Health and Nutrition Examination Survey. The purpose of this study was to examine the associations of serum vitamins levels with BMD at hip. Given both serum vitamins and BMD at hip were collected only in the third National Health and Nutrition Examination Survey (NHANES III), this study only used the data of the NHANES III. The NHANES III is designed to assess the health and nutritional status of residents in the US, and focused on oversampling many groups within the U.S. population aged $\geq 2$ months. These oversampled groups included children aged 2 months to 5 years, persons over age 60 , Mexican-American persons, and non-Hispanic black persons. The NHANES III interview includes demographic, socioeconomic, dietary, and health-related questions. The examination component consists of medical, dental, and physiological measurements, as well as laboratory tests administered by highly trained medical personnel. The detailed description of the NHANES III was published elsewhere [17]. This study was approved by the Institutional Review Board of the National Center for Health Statistics NHANES. All participants gave their written informed consent.

\section{Study population}

The included criteria were as follows: who were with complete data of interesting variables, such as BMD, serum vitamins, and other covariates, such as physical index, health behaviors, and medical histories; and who aged $\geq 18$ years. The excluded criteria were as follows: who were with missing data in the interesting variables; or who had the history of bone diseases, such as arthritis and bone tumor. The flowchart is shown in Fig. 1.

All participants were interviewed to collect data, including demographic-socioeconomic index and healthrelated questions. Meanwhile, fasting blood samples were collected to assay the levels of serum vitamins.

\section{Measures}

Serum samples from participants aged $\geq 18$ years who were interviewed during phase II of this survey (1991-1994) in the NHANES III surplus sera project were used to assay serum vitamins concentrations including vitamins A, B9, B12, C, and E using the Quantaphase II Radioassay Kit (Bio-Rad) in the NHANES Laboratory of the Centers for Disease Control and Prevention. The coefficients of variation for vitamins $\mathrm{A}, \mathrm{B} 9, \mathrm{~B} 12, \mathrm{C}$, and $\mathrm{E}$ in the NHANES III were $6,7,6,4$, and 5\%, respectively. Furthermore, the intra-assay and inter-assay coefficients of variation were 5.2 and $2.5 \%$, respectively. Details of the detection method are accessible at the NHANES website [18]. Dual-energy X-ray absorptiometry by using Hologic QDR 4500A fan-beam densitometers was employed to measure BMD at hip, including femur neck and the total hip BMD. The coefficients of variation were 3.2 and $2.4 \%$, respectively. Details on the DXA examination protocol have been published elsewhere [13].

\section{Covariates}

Height and weight were measured according to the standardized protocol, and used to calculate body mass index. All participants were divided into the White and Black as race, Mexican-American and Not Hispanic as ethnicity, or Non-Hispanic white, NonHispanic black, and Mexican-American as raceethnicity. Urbanization classification based USDA Rural/Urban continuum codes was as follows: central counties or fringe counties of metro areas with $\geq 1$ million population were considered as urban region; all other areas were considered as rural region. Health behaviors, such as smoking status, alcohol consumption, and physical activity, as well as medical histories, such as history of hypertension, history of diabetes, and history of fracture, were collected using a valid questionnaire. 


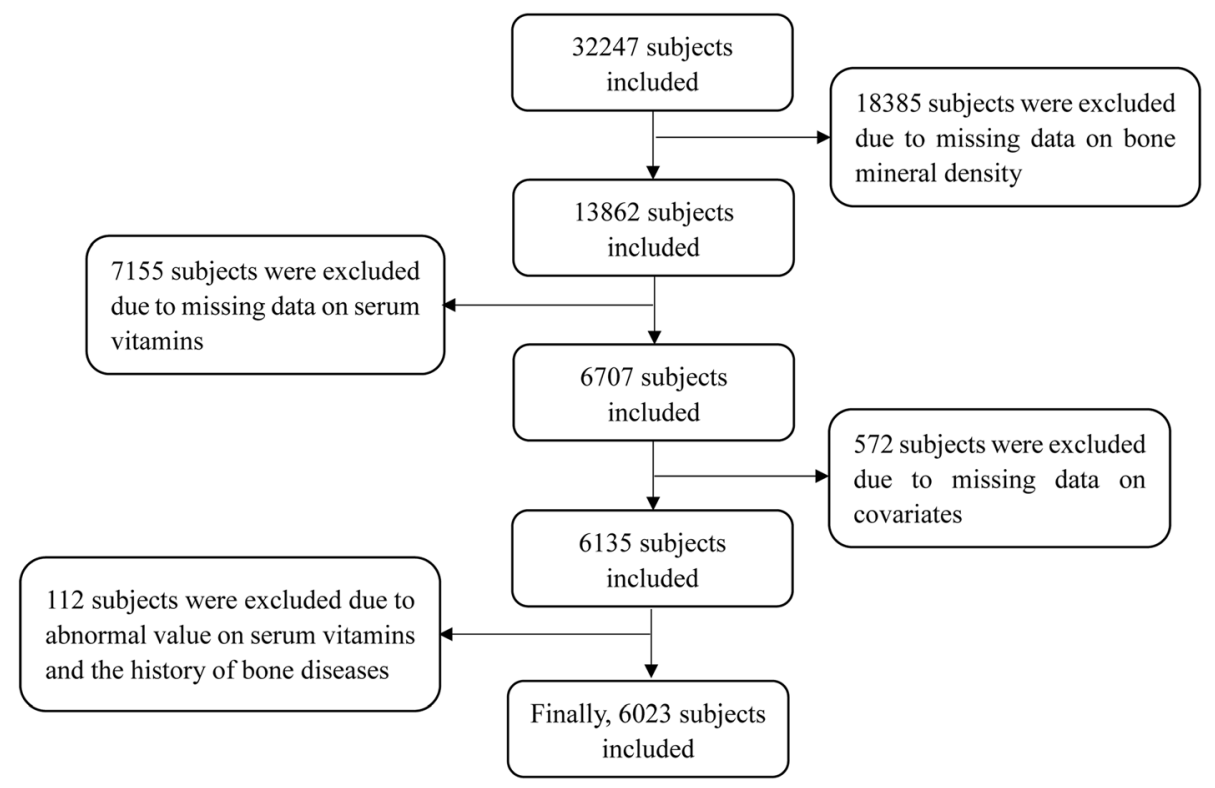

Fig. 1 The flowchart of this study design

\section{Statistical analysis}

Since all continuous variables were normal distribution, means and standard deviations were used to express the distributions of continuous variables. Categorical variables were described by frequencies and percentages. Linear regressions were employed to examine the associations of serum vitamins levels with BMD at different sites and obtain the regression coefficients and 95\% confidential intervals $(C I S)$. Furthermore, linear regressions were stratified by race, ethnicity, age, and sex. In order to correct the confounding effects of covariates, age, sex, body mass index, smoking, alcohol consumption, physical activity, years of school completed, living regions, race, ethnicity, history of hypertension, history of diabetes, history of fracture, and sample design weights were adjusted. All analyses were conducted using SAS 9.4 (SAS Institute Inc., Cary, NC, USA.). A two-tailed $P \leq 0.05$ indicated the statistical significance.

\section{Results}

\section{The characteristics of all participants}

There were 6023 participants included in the final analysis. The mean of age was 48.62 years. The averages of serum vitamins levels were $1.98 \mu \mathrm{mol} / \mathrm{L}$ for vitamin A, $15.67 \mathrm{nmol} / \mathrm{L}$ for folate, $358.75 \mathrm{pmol} / \mathrm{L}$ for vitamin B12, $41.25 \mathrm{mmol} / \mathrm{L}$ for vitamin $\mathrm{C}$, and $26.60 \mu \mathrm{mol} / \mathrm{L}$ for vitamin $\mathrm{E}$. And the averages of BMD were $0.83 \mathrm{~g} / \mathrm{cm}^{2}$ and $0.95 \mathrm{~g} / \mathrm{cm}^{2}$ at femur neck and the total hip, respectively. There were 2686 males, accounting for $44.60 \%$. All characteristics are shown in Table 1.

\section{The associations of serum vitamins levels with BMD}

Table 2 displays the associations of serum vitamins levels with BMD. Serum folate level was positively associated with BMD at femur neck and the total hip $(P=$ 0.035 and $0.010 ; \beta=0.00024$ and 0.00023 ; and $95 \% \mathrm{CI}$ : 0.00002-0.00051 and 0.00005-0.00051, respectively). Similarly, serum vitamins $A$ and $C$ levels were significantly associated with BMD at femur neck and the total hip (vitamin A: $P=0.010$ and $0.006 ; \beta=0.00749$ and 0.00837 ; and 95\% CI: $0.00176-0.01322$ and $0.00240-$ 0.01433 , respectively) and (vitamin $C$ : all $P<0.001 ; \beta=$ 0.00026 and 0.00025 ; and 95\% CI: 0.00012-0.00039 and 0.00011-0.00039, respectively). No significant associations of serum vitamins B12 and E levels with BMD were observed.

\section{The associations of serum vitamins levels with BMD stratified by race and ethnicity}

The results stratified by race are presented in Table 3. The associations of serum vitamins B12 and E levels with BMD were consistent with those of the total population. However, there were positive associations of serum folate level with BMD at femur neck and the total hip in the Black participants $(P=0.013$ and $0.018 ; \beta=$ 0.00086 and 0.00082 ; and $95 \%$ CI: $0.00018-0.00154$ and 0.00014-0.00151, respectively) but not in the White participants. Significant associations of serum vitamin A level with BMD were observed at femur neck in the White participants $(P=0.012)$ and at the total region in the Black participants $(P=0.024)$. Serum vitamin $C$ was associated with BMD at femur neck both in the White and Black populations $(P=0.011$ and 0.017 , respectively), 
Table 1 The characteristics of all participants $(N=6023)$

\begin{tabular}{|c|c|c|}
\hline Characteristics & Mean/Frequency & Standard deviation/Percentage (\%) \\
\hline Age (years) & 48.62 & 18.92 \\
\hline Years of school completed (years) & 11.12 & 3.85 \\
\hline Serum folate (nmol/L) & 15.67 & 12.44 \\
\hline Serum vitamin B12 (pmol/L) & 358.75 & 146.83 \\
\hline Serum vitamin C (mmol/L) & 41.25 & 24.65 \\
\hline Serum vitamin $A(\mu \mathrm{mol} / \mathrm{L})$ & 1.98 & 0.57 \\
\hline Serum vitamin $E(\mu \mathrm{mol} / \mathrm{L})$ & 26.60 & 11.39 \\
\hline Body mass index $\left(\mathrm{kg} / \mathrm{m}^{2}\right)$ & 27.42 & 5.66 \\
\hline Bone mineral density of femur neck $\left(\mathrm{g} / \mathrm{cm}^{2}\right)$ & 0.83 & 0.17 \\
\hline Bone mineral density of total region $\left(\mathrm{g} / \mathrm{cm}^{2}\right)$ & 0.95 & 0.18 \\
\hline \multicolumn{3}{|l|}{ Sex } \\
\hline Male & 2686 & 44.60 \\
\hline Female & 3337 & 55.40 \\
\hline \multicolumn{3}{|l|}{ Race } \\
\hline White & 4164 & 69.13 \\
\hline Black & 1859 & 30.87 \\
\hline \multicolumn{3}{|l|}{ Ethnicity } \\
\hline Mexican-American & 1484 & 24.64 \\
\hline Not Hispanic & 4539 & 75.36 \\
\hline \multicolumn{3}{|l|}{ Race-ethnicity } \\
\hline Non-Hispanic white & 2687 & 44.61 \\
\hline Non-Hispanic black & 1852 & 30.75 \\
\hline Mexican-American & 1484 & 24.64 \\
\hline \multicolumn{3}{|l|}{ Smoking } \\
\hline No & 4479 & 74.36 \\
\hline Yes & 1544 & 25.64 \\
\hline \multicolumn{3}{|l|}{ Alcohol consumption } \\
\hline No & 5230 & 86.83 \\
\hline Yes & 793 & 13.17 \\
\hline \multicolumn{3}{|l|}{ Living regionss } \\
\hline Urban & 2825 & 46.90 \\
\hline Rural & 3198 & 53.10 \\
\hline \multicolumn{3}{|l|}{ Physical activity } \\
\hline No & 3512 & 58.31 \\
\hline Yes & 2511 & 41.69 \\
\hline \multicolumn{3}{|l|}{ History of fracture } \\
\hline No & 5489 & 91.13 \\
\hline Yes & 534 & 8.87 \\
\hline \multicolumn{3}{|l|}{ History of hypertension } \\
\hline No & 2490 & 41.34 \\
\hline Yes & 3533 & 58.66 \\
\hline \multicolumn{3}{|l|}{ History of diabetes } \\
\hline No & 1104 & 18.33 \\
\hline Yes & 4919 & 81.67 \\
\hline
\end{tabular}


Table 2 The associations of serum vitamins levels with bone mineral density at different sites $(N=6023)^{a}$

\begin{tabular}{|c|c|c|c|c|}
\hline Sites & Serum vitamins & $\beta$ & $95 \% \mathrm{Cl}$ & $P$ \\
\hline \multicolumn{5}{|c|}{ Femur neck } \\
\hline & Serum folate & 0.00024 & $0.00002-0.00051$ & 0.035 \\
\hline & Serum vitamin A & 0.00749 & $0.00176-0.01322$ & 0.010 \\
\hline & Serum vitamin B12 & 0.00001 & $-0.00002-0.00002$ & 0.971 \\
\hline & Serum vitamin $C$ & 0.00026 & $0.00012-0.00039$ & $<0.001$ \\
\hline & Serum vitamin $\mathrm{E}$ & 0.00013 & $-0.00016-0.00043$ & 0.383 \\
\hline \multicolumn{5}{|c|}{ Total region } \\
\hline & Serum folate & 0.00023 & $0.00005-0.00051$ & 0.010 \\
\hline & Serum vitamin A & 0.00837 & $0.00240-0.01433$ & 0.006 \\
\hline & Serum vitamin B12 & 0.00001 & $-0.00001-0.00003$ & 0.461 \\
\hline & Serum vitamin $C$ & 0.00025 & $0.00011-0.00039$ & $<0.001$ \\
\hline & Serum vitamin $\mathrm{E}$ & 0.00028 & $-0.00003-0.00058$ & 0.077 \\
\hline
\end{tabular}

In all sites, age, sex, body mass index, years of school completed, race, ethnicity, living regions, smoking, alcohol consumption, physical activity, history of hypertension, history of diabetes, history of fracture, and sample design weights were adjusted

and at total region only in the White population $(P=$ 0.007).

As stratified by ethnicity, both in the MexicanAmerican and Not Hispanic participants, there were no significant associations of serum vitamins $\mathrm{B} 12$ and $\mathrm{E}$ levels with BMD, which were comparable with the total results. There were positive associations of serum folate, vitamin $\mathrm{A}$, and vitamin $\mathrm{C}$ levels with $\mathrm{BMD}$ at femur neck $(P=0.047,0.001$, and 0.001 , respectively) and the total hip $(P=0.039,0.001$, and $<0.001$, respectively) in the Not Hispanic participants but not in MexicanAmerican participants (Table 4).
The results stratified by age are shown in Table 5 . Significant associations of serum vitamin $C$ level with BMD at femur neck and the total hip were observed both in the 20-49 years and 50-90 years groups $(P=0.003$, $0.019,0.013$, and 0.025 , respectively). There were positive associations of serum folate level with BMD at femur neck and the total hip only in the 50-90 years group $(P=0.022$ and $0.041 ; \beta=0.00018$ and 0.00013 ; and 95\% CI: 0.00007-0.00028 and 0.00004-0.00023, respectively). Similarly, serum vitamin A level was significantly associated with BMD in the 50-90 years group at femur neck $(P=0.043 ; \beta=0.00834$; and $95 \% C I$ : $0.00026-$ $0.01642)$ and at the total region $(P=0.042 ; \beta=0.00913$; and 95\% CI: 0.00032-0.01793). In line with the total results, there were no significant associations of serum vitamins B12 and E levels with BMD both in the 20-49 years and 50-90 years groups.

Table 6 presents the associations of serum vitamins levels with BMD at femur neck and the total hip stratified by sex. In males, there were positively associations of serum folate, vitamin $\mathrm{A}$ and $\mathrm{C}$ levels with BMD at both femur neck $(P=0.014,0.004$, and $0.046 ; \beta=0.00040,0.01365$, and 0.00022 ; and $95 \%$ CI: $\quad 0.00010-0.00089, \quad 0.00431-0.02298$, and $0.00001-0.00044$, respectively) and total region $(P=$ $0.010,<0.001$, and $0.016 ; \beta=0.00042,0.01709$, and 0.00028 ; and $95 \%$ CI: $0.00018-0.00092,0.00761-$ 0.02657 , and $0.00005-0.00050$, respectively). However, in females, only serum vitamin $C$ was associated with BMD at femur neck and total region $(P=$ 0.001 and $0.002 ; \beta=0.00029$, and 0.00028 ; and $95 \%$ CI: $\quad 0.00012-0.00045$ and $0.00010-0.00045$, respectively).

Table 3 The associations of serum vitamins levels with bone mineral density at different sites stratified by race ${ }^{a}$

\begin{tabular}{|c|c|c|c|c|c|c|c|}
\hline \multirow[t]{2}{*}{ Sites } & \multirow[t]{2}{*}{ Serum vitamins } & \multicolumn{3}{|c|}{ White $(N=4164)$} & \multicolumn{3}{|c|}{ Black $(N=1859)$} \\
\hline & & $\bar{\beta}$ & $95 \% \mathrm{Cl}$ & $P$ & $\bar{\beta}$ & $95 \% \mathrm{Cl}$ & $P$ \\
\hline \multicolumn{8}{|c|}{ Femur neck } \\
\hline & Serum folate & 0.00007 & $-0.00022-0.00035$ & 0.636 & 0.00086 & $0.00018-0.00154$ & 0.013 \\
\hline & Serum vitamin $A$ & 0.00590 & $0.00075-0.01258$ & 0.012 & 0.01020 & $-0.00094-0.02134$ & 0.073 \\
\hline & Serum vitamin B12 & 0.00001 & $-0.00002-0.00003$ & 0.715 & -0.00001 & $-0.00005-0.00003$ & 0.612 \\
\hline & Serum vitamin $C$ & 0.00019 & $0.00004-0.00034$ & 0.011 & 0.00036 & $0.00006-0.00066$ & 0.017 \\
\hline & Serum vitamin E & 0.00022 & $-0.00009-0.00053$ & 0.158 & -0.00049 & $-0.00126-0.00029$ & 0.218 \\
\hline \multicolumn{8}{|c|}{ Total region } \\
\hline & Serum folate & 0.00006 & $-0.00024-0.00036$ & 0.690 & 0.00082 & $0.00014-0.00151$ & 0.018 \\
\hline & Serum vitamin A & 0.00511 & $-0.00193-0.01215$ & 0.155 & 0.01297 & $0.00171-0.02423$ & 0.024 \\
\hline & Serum vitamin B12 & 0.00001 & $-0.00002-0.00004$ & 0.474 & 0.00001 & $-0.00004-0.00004$ & 0.954 \\
\hline & Serum vitamin $C$ & 0.00021 & $0.00006-0.00037$ & 0.007 & 0.00029 & $-0.00001-0.00059$ & 0.061 \\
\hline & Serum vitamin $E$ & 0.00030 & $-0.00003-0.00062$ & 0.076 & -0.00005 & $-0.00083-0.00073$ & 0.903 \\
\hline
\end{tabular}

In all sites, age, sex, body mass index, years of school completed, ethnicity, living regions, smoking, alcohol consumption, physical activity, history of hypertension, history of diabetes, history of fracture, and sample design weights were adjusted 
Table 4 The associations of serum vitamins levels with bone mineral density at different sites stratified by ethnicity ${ }^{a}$

\begin{tabular}{|c|c|c|c|c|c|c|c|}
\hline \multirow[t]{2}{*}{ Sites } & \multirow[t]{2}{*}{ Serum vitamins } & \multicolumn{3}{|c|}{ Mexican-American $(N=1484)$} & \multicolumn{3}{|c|}{ Not Hispanic $(N=4539)$} \\
\hline & & $\beta$ & $95 \% \mathrm{Cl}$ & $P$ & $\beta$ & $95 \% \mathrm{Cl}$ & $P$ \\
\hline \multicolumn{8}{|c|}{ Femur neck } \\
\hline & Serum folate & 0.00003 & $-0.00067-0.00073$ & 0.925 & 0.00027 & $0.00002-0.00057$ & 0.047 \\
\hline & Serum vitamin $A$ & -0.00740 & $-0.01947-0.00467$ & 0.229 & 0.01132 & $0.00478-0.01787$ & 0.001 \\
\hline & Serum vitamin B12 & 0.00003 & $-0.00001-0.00007$ & 0.157 & -0.00001 & $-0.00003-0.00002$ & 0.501 \\
\hline & Serum vitamin $C$ & 0.00015 & $-0.00013-0.00044$ & 0.290 & 0.00027 & $0.00012-0.00042$ & 0.001 \\
\hline & Serum vitamin $\mathrm{E}$ & 0.00020 & $-0.00039-0.00080$ & 0.500 & 0.00012 & $-0.00022-0.00045$ & 0.504 \\
\hline \multicolumn{8}{|c|}{ Total region } \\
\hline & Serum folate & -0.00032 & $-0.00104-0.00039$ & 0.379 & 0.00032 & $0.00002-0.00063$ & 0.039 \\
\hline & Serum vitamin $A$ & -0.00134 & $-0.01370-0.01101$ & 0.831 & 0.01115 & $0.00430-0.01799$ & 0.001 \\
\hline & Serum vitamin B12 & 0.00004 & $-0.00001-0.00008$ & 0.086 & -0.00001 & $-0.00003-0.00003$ & 0.960 \\
\hline & Serum vitamin $C$ & 0.00001 & $-0.00025-0.00034$ & 0.766 & 0.00029 & $0.00013-0.00045$ & $<0.001$ \\
\hline & Serum vitamin $\mathrm{E}$ & 0.00022 & $-0.00039-0.00083$ & 0.474 & 0.00032 & $-0.00003-0.00067$ & 0.077 \\
\hline
\end{tabular}

${ }^{a}$ In all sites, age, sex, body mass index, years of school completed, race, living regions, smoking, alcohol consumption, physical activity, history of hypertension, history of diabetes, history of fracture, and sample design weights were adjusted

\section{Discussion}

This study aimed to investigate the associations of serum vitamins levels with BMD at hip in US adults. The results suggested that serum folate, vitamins $A$ and $C$ levels were positively associated with BMD at femur neck and the total hip. Furthermore, significant associations of serum vitamin $C$ level with BMD were fully observed both in the White and Black participants. However, the associations of serum folate, vitamins A and $C$ levels with BMD were fully observed only in the Not Hispanic, 50-90 years, and male participants.

The results implied that serum folate level was positively associated with BMD at femur neck and the total hip, which was consistent with previous studies $[6,17]$. Like tetrahydrobiopterin, folate was a cofactor for the enzyme of nitric oxide synthase, which can promote the maintenance of bone density by helping to preserve optimal nitric oxide synthase activity in the bone cells [19]. Furthermore, choline played important role in skeletal muscle through fat and protein metabolism, inflammation, and autophagy [20]. Adequate serum folate level could properly modulate fat and protein metabolism, which in turn decrease fatty acid synthesis and promote muscle growth and function [20]. On the other hand, adequate dietary intake of folate could counteract inflammation, apoptosis, and autophagy via promoting

Table 5 The associations of serum vitamins levels with bone mineral density at different sites stratified by age $\mathrm{e}^{\mathrm{a}}$

\begin{tabular}{|c|c|c|c|c|c|c|c|}
\hline \multirow[t]{2}{*}{ Sites } & \multirow[t]{2}{*}{ Serum vitamins } & \multicolumn{3}{|c|}{$20-49$ years $(N=3364)$} & \multicolumn{3}{|c|}{$50-90$ years $(N=2600)$} \\
\hline & & $\bar{\beta}$ & $95 \% \mathrm{Cl}$ & $P$ & $\bar{\beta}$ & $95 \% \mathrm{Cl}$ & $P$ \\
\hline \multicolumn{8}{|c|}{ Femur neck } \\
\hline & Serum folate & -0.00020 & $-0.00072-0.00032$ & 0.445 & 0.00018 & $0.00007-0.00028$ & 0.022 \\
\hline & Serum vitamin A & 0.00252 & $-0.00666-0.01170$ & 0.590 & 0.00834 & $0.00026-0.01642$ & 0.043 \\
\hline & Serum vitamin B12 & -0.00001 & $-0.00004-0.00003$ & 0.744 & 0.00002 & $-0.00001-0.00004$ & 0.304 \\
\hline & Serum vitamin $C$ & 0.00032 & $0.00011-0.00054$ & 0.003 & 0.00013 & $0.00006-0.00022$ & 0.019 \\
\hline & Serum vitamin $\mathrm{E}$ & -0.00097 & $-0.00154-0.00040$ & 0.101 & 0.00009 & $-0.00028-0.00045$ & 0.639 \\
\hline \multicolumn{8}{|c|}{ Total region } \\
\hline & Serum folate & -0.00005 & $-0.00055-0.00045$ & 0.836 & 0.00013 & $0.00004-0.00023$ & 0.041 \\
\hline & Serum vitamin A & 0.00724 & $-0.00165-0.01612$ & 0.111 & 0.00913 & $0.00032-0.01793$ & 0.042 \\
\hline & Serum vitamin B12 & -0.00001 & $-0.00003-0.00003$ & 0.923 & 0.00003 & $-0.00001-0.00007$ & 0.062 \\
\hline & Serum vitamin $C$ & 0.00026 & $0.00005-0.00047$ & 0.013 & 0.00023 & $0.00003-0.00044$ & 0.025 \\
\hline & Serum vitamin $\mathrm{E}$ & -0.00023 & $-0.00079-0.00032$ & 0.411 & 0.00019 & $-0.00021-0.00059$ & 0.358 \\
\hline
\end{tabular}

In all sites, sex, body mass index, years of school completed, race, ethnicity, living regions, smoking, alcohol consumption, physical activity, history of hypertension, history of diabetes, history of fracture, and sample design weights were adjusted 
Table 6 The associations of serum vitamins levels with bone mineral density at different sites stratified by sex ${ }^{a}$

\begin{tabular}{|c|c|c|c|c|c|c|c|}
\hline \multirow[t]{2}{*}{ Sites } & \multirow[t]{2}{*}{ Serum vitamins } & \multicolumn{3}{|c|}{ Males $(N=2686)$} & \multicolumn{3}{|c|}{ Females $(N=3337)$} \\
\hline & & $\beta$ & $95 \% \mathrm{Cl}$ & $P$ & $\beta$ & $95 \% \mathrm{Cl}$ & $P$ \\
\hline \multicolumn{8}{|c|}{ Femur neck } \\
\hline & Serum folate & 0.00040 & $0.00010-0.00089$ & 0.014 & 0.00016 & $-0.00015-0.00047$ & 0.299 \\
\hline & Serum vitamin A & 0.01365 & $0.00431-0.02298$ & 0.004 & 0.00298 & $-0.00421-0.01018$ & 0.417 \\
\hline & Serum vitamin B12 & -0.00001 & $-0.00005-0.00002$ & 0.432 & 0.00001 & $-0.00001-0.00004$ & 0.304 \\
\hline & Serum vitamin C & 0.00022 & $0.00001-0.00044$ & 0.046 & 0.00029 & $0.00012-0.00045$ & 0.001 \\
\hline & Serum vitamin $\mathrm{E}$ & -0.00007 & $-0.00054-0.00040$ & 0.773 & 0.00030 & $-0.00008-0.00068$ & 0.118 \\
\hline \multicolumn{8}{|c|}{ Total region } \\
\hline & Serum folate & 0.00042 & $0.00018-0.00092$ & 0.010 & 0.00015 & $-0.00018-0.00048$ & 0.365 \\
\hline & Serum vitamin A & 0.01709 & $0.00761-0.02657$ & $<0.001$ & 0.00316 & $-0.00442-0.01073$ & 0.414 \\
\hline & Serum vitamin B12 & 0.00001 & $-0.00003-0.00004$ & 0.728 & 0.00002 & $-0.00001-0.00005$ & 0.191 \\
\hline & Serum vitamin C & 0.00028 & $0.00005-0.00050$ & 0.016 & 0.00028 & $0.00010-0.00045$ & 0.002 \\
\hline & Serum vitamin $\mathrm{E}$ & 0.00029 & $-0.00019-0.00077$ & 0.232 & 0.00037 & $-0.00002-0.00077$ & 0.065 \\
\hline
\end{tabular}

${ }^{a}$ In all sites, age, body mass index, years of school completed, race, ethnicity, living regions, smoking, alcohol consumption, physical activity, history of hypertension, history of diabetes, history of fracture, and sample design weights were adjusted

intercellular homeostasis [20]. Thus, serum folate indirectly modulated bone metabolism via bone muscle cross-talk.

Several epidemiological studies reported that a higher of serum vitamin A level could improve bone health, which was in line with the findings of this study [21, 22]. The possible mechanisms might be summarized as follows: First, vitamin A could indirectly improve bone health by restraining the excessive secretion of parathyroid hormone, a higher level of which was suggested to result in poor bone health [23, 24]. Second, vitamin A can promote bone growth by means of affecting the growth hormone and stimulating the production of insulin-like growth factor 1 , which are conducive to bone health [25]. Third, oxidative stress and reactive oxygen species can promote the development of osteoporosis. Whilst vitamin A has a property of against them. Therefore, in this respect, vitamin A was associated with a better BMD [26, 27].

In this study, serum vitamin $C$ level was associated with a better BMD, which was consistent with previous studies $[28,29]$. It was confirmed that vitamin $C$ involved in the collagen synthesis and can especially stimulate type I and III collagen synthesis [30,31]. Furthermore, since vitamin $\mathrm{C}$ is essential for osteoblast genes, there was an active effect of vitamin $\mathrm{C}$ on bone formation by influencing expression of osteoblast genes and attenuating the loss of osteoblast differentiation markers [29]. Therefore, it was reasonable that vitamin $\mathrm{C}$ might improve bone health. In this study, there were no significant associations of serum vitamins B12 and E levels with BMD, which was consistent with previous studies [32, 33]. The potential mechanisms of serum folate, vitamins $\mathrm{A}$ and $\mathrm{C}$ levels on bone health are presented in Fig. 2.
Furthermore, it implied that race-ethnicity could modify the associations of serum vitamins levels with BMD in this study. Especially, serum folate level was associated with BMD at three site only in the Black participants. Significant associations of serum folate, vitamins A and C levels with BMD were observed only in the Not Hispanic participants. Many studies have reported that the Black subjects exhibited higher BMD than the White subjects at the femoral neck [14, 16, 34]. Meanwhile, a previous study declared that a lower hip strength was found in the Hispanic subjects compared with the White or Black subjects [35]. Furthermore, there were significant differences in the weight, body mass index, lean mass, and fat mass across races/ ethnicities, which could contribute to the differences of BMD [36]. Furthermore, the similar study from the NHANES III reported that the Mexican-America women had a higher femoral neck BMD and shorter hip axis length compared with the non-Hispanic White women in the USA [37]. Therefore, it was feasible that the race-ethnicity indeed affected the associations of serum vitamins levels with BMD.

In this study, it was found that there were positively associations of serum folate, vitamins $\mathrm{A}$ and $\mathrm{C}$ levels with BMD, especially in the 50-90 years and male population. The possible reasons might be that the nutrition status was worse in the older population than that in the young population. The prevalence of malnutrition and vitamins $\mathrm{A}$ and $\mathrm{C}$ deficiency significantly increased over age [38]. Therefore, the effects of serum vitamins levels on BMD might be more pronounced in the older population. Furthermore, serum vitamins levels and BMD in the elderly males were significantly higher than that in females, which might explain the sex difference in the 


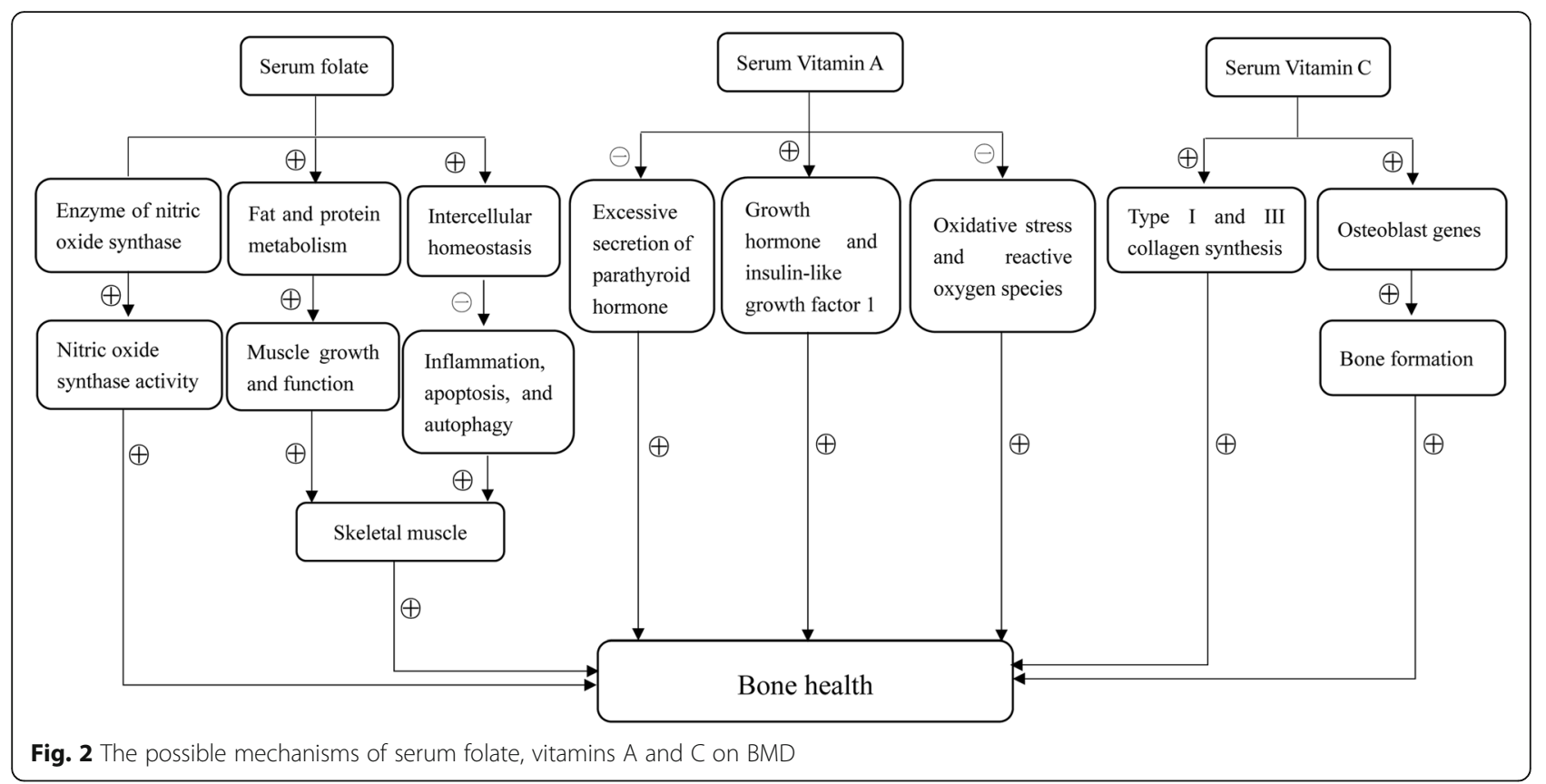

associations of serum folate, vitamins $\mathrm{A}$ and $\mathrm{C}$ levels with BMD [38, 39].

\section{Strengths and limitations}

There were some strengths in this study. First, this study comprehensively evaluated the associations of serum vitamins levels with BMD at hip in the US adults. Furthermore, some major covariates were adjusted in this study, such as race/ ethnicity, history of fracture, BMI and sample design weights. Therefore, this study would provide accurate evidence on the associations of serum vitamins levels with BMD. Second, differing from other studies to use dietary vitamins intake or supplement, serum vitamins levels were used in this study. Given the complicated relationship between dietary vitamins intake and serum level, serum vitamins levels seemed to better reflect the biological availability and activity of vitamins. Third, this study further examined the modified effect of race and ethnicity on the associations of serum vitamins levels with BMD. As a result, it would provide a better understanding on the important role of race-ethnicity in the associations of serum vitamins levels with BMD.

However, there were some limitations to be stated. First, this study was based on the NHANES III, in which the study population were restricted to the US subjects. Furthermore, the population of this study were relatively young. Therefore, it should be cautious when generalizing the results to older populations or populations outside the USA. Second, this study was a cross-sectional study, which was poor to examine the causal relationship between serum vitamins levels and BMD. A well designed cohort study should be conducted to confirm the findings of this study in the future. Third, since the complicated backgrounds of different races or ethnicities, the mechanisms of race/ ethnicity modifying the associations of serum vitamins levels with BMD failed to be fully explained in this study.

\section{Conclusions}

Serum folate, vitamins A and C levels were positively associated with BMD at femur neck and the total hip. Furthermore, age, sex, and race/ ethnicity modified the associations of serum vitamins levels with BMD. Significant associations of serum vitamins levels with BMD were fully observed in the Not Hispanic, 50-90 years, and male participants. Therefore, this study would provide additional evidence to improve bone health, improve understanding the role of age, sex, and race/ ethnicity in the associations of serum vitamins levels with BMD, and provide suggestions in public health interventions.

\section{Abbreviations \\ BMD: Bone mineral density; NHANES III: The third National Health and Nutrition Examination Survey:; Cls: Confidential intervals}

\section{Acknowledgements}

We thank the third National Health and Nutrition Examination Survey for the permission for use of the data of the NHANES III.

\section{Authors' contributions}

Xiang Li analyzed the data, interpreted the results, and wrote the draft. And Xun Liu contributed to the concept and design and edited the draft. All authors read and approved the final manuscript.

Funding

None. 


\section{Availability of data and materials}

The datasets analyzed during the current study are available in the website of the NHANES: https://www.cdc.gov/nchs/index.htm.

\section{Ethics approval and consent to participate}

This study was approved by the Institutional Review Board of the National Center for Health Statistics NHANES. All participants gave their written informed consent.

\section{Consent for publication}

Not applicable.

\section{Competing interests}

The authors declare that they have no conflict of interest.

\section{Author details}

'Department of Orthopedics, Tianjin Fifth Central Hospital, Tianjin 300450, China. ${ }^{2}$ Department of Ultrasonics, Tianjin Fifth Central Hospital, No 41

Zhejiang Road, Tianjin 300450, China.

Received: 13 December 2020 Accepted: 8 January 2021

Published online: 04 February 2021

\section{References}

1. Cummings SR, Melton LJ. Epidemiology and outcomes of osteoporotic fractures. Lancet. 2002;359(9319):1761-7.

2. Lippuner K, Golder M, Greiner R. Epidemiology and direct medical costs of osteoporotic fractures in men and women in Switzerland. Osteoporos Int. 2005;16(Suppl 2):S8-S17.

3. Johnell O, Kanis JA. An estimate of the worldwide prevalence and disability associated with osteoporotic fractures. Osteoporos Int. 2006;17(12):1726-33.

4. Wright NC, Saag KG, Dawson-Hughes B, Khosla S, Siris ES. The impact of the new National Bone Health Alliance (NBHA) diagnostic criteria on the prevalence of osteoporosis in the USA. Osteoporos Int. 2017;28(4):1225-32.

5. Burge R, Dawson-Hughes B, Solomon DH, Wong JB, King A, Tosteson A. Incidence and economic burden of osteoporosis-related fractures in the United States, 2005-2025. J Bone Miner Res. 2007:22(3):465-75.

6. Herrmann M, Umanskaya N, Traber L, Schmidt-Gayk H, Menke W, Lanzer G, et al. The effect of B-vitamins on biochemical bone turnover markers and bone mineral density in osteoporotic patients: a 1-year double blind placebo controlled trial. Clin Chem Lab Med. 2007:45(12):1785-92.

7. Genuis SJ, Schwalfenberg GK. Picking a bone with contemporary osteoporosis management: nutrient strategies to enhance skeletal integrity. Clin Nutr. 2007:26(2):193-207.

8. Ilesanmi-Oyelere BL, Brough L, Coad J, Roy N, Kruger MC. The Relationship between Nutrient Patterns and Bone Mineral Density in Postmenopausal Women. Nutrients. 2019;11(6):1262.

9. Denova-Gutierrez E, Mendez-Sanchez L, Munoz-Aguirre P, Tucker KL, Clark P. Dietary Patterns, Bone Mineral Density, and Risk of Fractures: A Systematic Review and Meta-Analysis. Nutrients. 2018;10(12):1922.

10. Mott A, Bradley T, Wright K, Cockayne ES, Shearer MJ, Adamson J, et al. Effect of vitamin $\mathrm{K}$ on bone mineral density and fractures in adults: an updated systematic review and meta-analysis of randomised controlled trials. Osteoporos Int. 2019;30(8):1543-59.

11. Ahmadieh $\mathrm{H}$, Arabi A. Vitamins and bone health: beyond calcium and vitamin D. Nutr Rev. 2011;69(10):584-98.

12. Zhang H, Tao X, Wu J. Association of homocysteine, vitamin B12, and folate with bone mineral density in postmenopausal women: a meta-analysis. Arch Gynecol Obstet. 2014;289(5):1003-9.

13. Bailey RL, Looker AC, Lu Z, Fan R, Eicher-Miller HA, Fakhouri TH, et al. Bvitamin status and bone mineral density and risk of lumbar osteoporosis in older females in the United States. Am J Clin Nutr. 2015;102(3):687-94.

14. Shin MH, Zmuda JM, Barrett-Connor E, Sheu Y, Patrick AL, Leung PC, et al. Race/ethnic differences in associations between bone mineral density and fracture history in older men. Osteoporos Int. 2014;25(3):837-45.

15. Fang J, Freeman $\mathrm{R}$, Jeganathan $\mathrm{R}$, Alderman $\mathrm{MH}$. Variations in hip fracture hospitalization rates among different race/ethnicity groups in New York City. Ethn Dis. 2004;14(2):280-4.

16. Wright NC, Saag KG, Curtis JR, Smith WK, Kilgore ML, Morrisey MA, et al. Recent trends in hip fracture rates by race/ethnicity among older US adults. J Bone Miner Res. 2012;27(11):2325-32.
17. Morris MS, Jacques PF, Selhub J. Relation between homocysteine and Bvitamin status indicators and bone mineral density in older Americans. Bone. 2005;37(2):234-42.

18. CDC NCfHSa. Hyattsville (MD): National Health and Nutrition Examination Survey: NHANES III Survey Methods and Analytic Guidelines [Internet] [Available from: https://wwwn.cdc.gov/nchs/nhanes/nhanes3/ SurveyMethods.aspx.

19. McCarty MF. Supplemental arginine and high-dose folate may promote bone health by supporting the activity of endothelial-type nitric oxide synthase in bone. Med Hypotheses. 2005;64(5):1030-3.

20. Moretti A, Paoletta M, Liguori S, Bertone M, Toro G, lolascon G. Choline: An Essential Nutrient for Skeletal Muscle. Nutrients. 2020;12(7):2144.

21. Maggio D, Polidori MC, Barabani M, Tufi A, Ruggiero C, Cecchetti R, et al. Low levels of carotenoids and retinol in involutional osteoporosis. Bone. 2006;38(2):244-8.

22. de Jonge EA, Kiefte-de Jong JC, Campos-Obando N, Booij L, Franco OH, Hofman A, et al. Dietary vitamin a intake and bone health in the elderly: the Rotterdam study. Eur J Clin Nutr. 2015;69(12):1360-8.

23. Pluijm SM, Visser M, Smit JH, Popp-Snijders C, Roos JC, Lips P. Determinants of bone mineral density in older men and women: body composition as mediator. J Bone Miner Res. 2001;16(11):2142-51.

24. Paik JM, Farwell WR, Taylor EN. Demographic, dietary, and serum factors and parathyroid hormone in the National Health and nutrition examination survey. Osteoporos In. 2012;23(6):1727-36.

25. Savaris VDL, Souza C, Wachholz L, Broch J, Polese C, Carvalho PLO, et al. Interactions between lipid source and vitamin a on broiler performance, blood parameters, fat and protein deposition rate, and bone development. Poult Sci. 2021;100(1):174-85.

26. Sanchez-Rodriguez MA, Ruiz-Ramos M, Correa-Munoz E, Mendoza-Nunez VM. Oxidative stress as a risk factor for osteoporosis in elderly Mexicans as characterized by antioxidant enzymes. BMC Musculoskelet Disord. 2007;8:124.

27. Wilson C. Bone: oxidative stress and osteoporosis. Nat Rev Endocrinol. 2014; 10(1):3.

28. Rondanelli M, Opizzi A, Perna S, Faliva MA. Update on nutrients involved in maintaining healthy bone. Endocrinol Nutr. 2013;60(4):197-210.

29. Aghajanian P, Hall S, Wongworawat MD, Mohan S. The roles and mechanisms of actions of vitamin C in bone: new developments. J Bone Miner Res. 2015;30(11):1945-55.

30. Carinci F, Pezzetti F, Spina AM, Palmieri A, Laino G, De Rosa A, et al. Effect of vitamin C on pre-osteoblast gene expression. Arch Oral Biol. 2005;50(5):481-96.

31. Abdullah M, Jamil RT, Attia FN. Vitamin C (Ascorbic Acid). Treasure Island (FL): StatPearls; 2020

32. Beyazit F, Pek E. Effects of vitamin B12, folate, uric acid, and serum biomarkers of inflammation on bone mineral density in postmenopausal women. Prz Menopauzalny. 2018;17(2):69-76.

33. Tennant KG, Leonard SW, Wong CP, Iwaniec UT, Turner RT, Traber MG. High-dietary alpha-Tocopherol or mixed Tocotrienols have no effect on bone mass, density, or turnover in male rats during skeletal maturation. J Med Food. 2017;20(7):700-8.

34. Peacock M, Buckwalter KA, Persohn S, Hangartner TN, Econs MJ, Hui S. Race and sex differences in bone mineral density and geometry at the femur. Bone. 2009:45(2):218-25.

35. Travison TG, Beck TJ, Esche GR, Araujo AB, McKinlay JB. Age trends in proximal femur geometry in men: variation by race and ethnicity. Osteoporos Int. 2008;19(3):277-87.

36. Berenson $A B$, Rahman M, Wilkinson $G$. Racial difference in the correlates of bone mineral content/density and age at peak among reproductive-aged women. Osteoporos Int. 2009;20(8):1439-49.

37. Looker AC, Orwoll ES, Johnston CC Jr, Lindsay RL, Wahner HW, Dunn WL, et al. Prevalence of low femoral bone density in older U.S. adults from NHANES III. J Bone Miner Res. 1997;12(11):1761-8.

38. Chen J, Hu YC, Yang C, Yun CF, Wang R, Mao DQ, et al. Study on vitamin a nutritional status of Chinese urban elderly residents in 2010-2012. Zhonghua Yu Fang Yi Xue Za Zhi. 2017:51(2):121-4.

39. Henry YM, Eastell R. Ethnic and gender differences in bone mineral density and bone turnover in young adults: effect of bone size. Osteoporos Int. 2000;11(6):512-7.

\section{Publisher's Note}

Springer Nature remains neutral with regard to jurisdictional claims in published maps and institutional affiliations. 